\title{
Improvement of the Sandell-Kolthoff reaction method (ammonium persulfate digestion) for the determination of iodine in urine samples
}

DOI 10.1515/cclm-2016-1094

Received December 2, 2016; accepted February 3, 2017

Keywords: inter-laboratory study; iodine; Sandell-Kolthoff reaction; urine samples.

To the Editor,

Iodine is an essential component of thyroid hormones; thus, its deficiency may impair growth and brain development at early stages of life and impair normal physical and mental activities development and lead to goiter later [1]. Iodine deficiency remains a worldwide-recognized problem, with $30 \%$ of the population living in areas with iodine-deficient soil [2] and over 2 billion individuals have insufficient iodine intake [3].

Among the several current methods used for iodine concentration determination in urine, several detection systems are available, namely spectrophotometric, potentiometric, and ICP-MS [4, 5]. The most commonly used methods for urinary iodine concentration measurement use the Sandell-Kolthoff reaction [6], with $34 \%$ of the overall methods (Supplemental Figure 1).

The method consists of urine digestion with chloric acid, ammonium persulfate or ashing, digestion, to eliminate interfering substances and release iodide, followed

*Corresponding author: Dr. Raquel B.R. Mesquita, CBQF - Escola Superior de Biotecnologia, Universidade Católica Portuguesa/Porto, R. Arquiteto Lobão Vital, Apartado 2511, 4202-401 Porto, Portugal, Phone: +351220428182, E-mail: rbmesquita@icbas.up.pt; ICBAS/ UP - Instituto de Ciências Biomédicas Abel Salazar, Universidade do Porto; and CBQF - Centro de Biotecnologia e Química Fina Laboratório Associado, Escola Superior de Biotecnologia, Universidade Católica Portuguesa/Porto, Porto, Portugal Ana Machado and Adriano A. Bordalo: ICBAS/UP - Instituto de Ciências Biomédicas Abel Salazar, Universidade do Porto, Porto, Portugal; and CIIMAR/CIMAR - Centro Interdisciplinar de Investigação Marinha e Ambiental, Universidade do Porto, Porto, Portugal Lurdes Lima: ICBAS/UP - Instituto de Ciências Biomédicas Abel Salazar, Universidade do Porto, Porto, Portugal by the Sandell-Kolthoff reaction [6]. Iodide catalyzes the reduction of the yellow-colored ceric(IV) ions by arsenic to colorless ceric(III) ions and elemental iodine, allowing the spectrophotometric detection of the color disappearance.

In this work, several parameters of the method were revisited in order to improve the robustness and reliability of the overall assessment. The number of steps was reduced and was standardized to reduce the probability of errors and contamination; the amount of reagents was minimized for a more cost-effective procedure with less waste production, i.e. greener approach; the sample volume was increased to have lower dilution factor with consequent increase in sensitivity.

The whole procedure was performed in duplicate given the limitation of the available thermo reactor (Spectroquant TR 20, Merck) of 24 wells; calibration curves with six standards (12 wells) and six samples also with two replicates (12 wells) per run were prepared.

For each calibration curve, different volumes of the iodine standard of $1 \mathrm{mg} \mathrm{I} / \mathrm{L}$ and water were measured directly to appropriate glass vials $(13 \times 100 \mathrm{~mm})$ to a final volume of $300 \mu \mathrm{L}$ (Supplemental Table 1). Then to each tube, $1 \mathrm{~mL}$ of ammonium persulfate solution was added and vortexed. The mixture was heated for $1 \mathrm{~h}$ at $100{ }^{\circ} \mathrm{C}$. After reaching room temperature, $2.5 \mathrm{~mL}$ of arsenious acid was added. It was reacted for $15 \mathrm{~min}$ prior to the addition of $300 \mu \mathrm{L}$ of cerium(IV) solution. Since it was imperative to measure the absorbance exactly $30 \mathrm{~min}$ after the addition of cerium(IV), intervals of $30 \mathrm{~s}$ between procedures were chosen. The used wavelength was $420 \mathrm{~nm}$, representing maximum absorbance of cerium(IV). The reaction with cerium(IV) is kinetic related, so in the former procedure, it is advisable that the interval between the addition cerium(IV) and the absorbance reading is exactly the same for samples and standards. Usually, it is recommended to add cerium(IV) to each sample/standard with 15- to 30-s intervals. The reading should be precisely $30 \mathrm{~min}$ after the addition. These guidelines are subject to different interpretations, inducing high variability in the attained results. Standardization of the cerium(IV) addition was 
achieved by adding the solution with 30-s interval to each analysis vials, and immediately after $30 \mathrm{~min}$ of the first addition, the absorbance was registered in $30 \mathrm{~s}$ intervals. This way, there was enough time to make the individual readings, ensuring exactly 30 -min reaction for each analysis vial (sample and standards).

The former procedure of the method required the preparation of two stock solutions (1000 mg I/L or $100 \mathrm{mg}$ $\mathrm{I} / \mathrm{L}$ and $10 \mathrm{mg} \mathrm{I} / \mathrm{L}$ or $1 \mathrm{mg} \mathrm{I} / \mathrm{L}$ ) obtained by dissolution of potassium iodate, together with the daily preparation of five working standards. This procedure largely increases the potential errors with dilution factor, and as the iodine solutions are not stable at concentrations below $1 \mathrm{mg} \mathrm{I} / \mathrm{L}$, there is degradation of the working standards during the working day.

One stock solution of $10 \mathrm{mg} \mathrm{I} / \mathrm{L}$ was maintained, which is stable for $6-12$ months if kept refrigerated $\left(4^{\circ} \mathrm{C}\right)$, in a dark plastic bottle. Dilution to $1 \mathrm{mg} \mathrm{I} / \mathrm{L}$ was freshly prepared, and no working standards were needed, different concentrations were obtained using different volumes (Supplemental Table 1). Therefore, one less dilution step was performed, and there was no concern with potential working day degradation as only one solution was prepared $(1 \mathrm{mg} \mathrm{I} / \mathrm{L})$ and immediately used. The sample volume was increased to $300 \mu \mathrm{L}$ (instead of the former $250 \mu \mathrm{L}$ ), decreasing the dilution factor from 5 to approximately 4 . This can be an advantage for samples with low iodine content.

As the aim was to verify the effectiveness of the improvement of all calibration parameters, namely the slope and the intercept, they were compared and not only the correlation factor [7]. Calibration curves were performed using the improved procedure $\left(\mathrm{A}=-6.43 \mathrm{mg} \mathrm{I} / \mathrm{L}+1.601 ; \mathrm{r}^{2}=0.996\right)$ and were compared to calibration curves performed with the former method $\left(A=-4.44 \mathrm{mg} \mathrm{I} / \mathrm{L}+1.341 ; \mathrm{r}^{2}=0.949\right)$
(Figure 1). An improvement in the sensitivity (45\%) and in the reproducibility $(70 \%)$ of the standard replicates was observed. Although the blank was always difficult to include in the calibration curve, it becomes much closer with the improved method, relative deviation to the intercept $-8 \%$ vs. $-41 \%$ in the former method.

The improved method robustness was also tested and compared to the former method. Several samples (\#115) were analyzed and the relative deviation calculated (Supplemental Figure 2). With the improved method, no samples with a relative deviation above $10 \%$ were found; a major improvement to the former method, where $20 \%$ of the samples presented relative deviations above $10 \%$ and $6 \%$ of the samples had relative deviation above $20 \%$.

The described method enabled the determination of iodine in a batchwise approach in approximately $2 \mathrm{~h}$ and $45 \mathrm{~min}$, being the number of samples dependent on the digester capacity.

The robustness of the procedure, previously observed with the relative standard deviation (RSD) of $<10 \%$ $(n=115)$, was also assessed with calibration curves. Assessment of the reproducibility was performed by two independent operators within one year of analysis and RSD of 7\% for the calibration slope and 3\% for the intercept were obtained. For the repeatability, the same operator performed several calibration curves within a month and RSD of $1 \%$ for the calibration slope and $2 \%$ for the intercept were obtained.

The overall reagent consumption per vial was $228 \mathrm{mg}$ of persulfate, $24 \mathrm{mg}$ of cerium(IV) ammonium sulfate, $25 \mathrm{mg}$ of arsenic trioxide, $62.5 \mathrm{mg}$ of sodium chloride, and $575 \mathrm{mg}$ of sulfuric acid.

The accuracy of the improved method was assessed by comparing the results obtained with an international

\section{A}

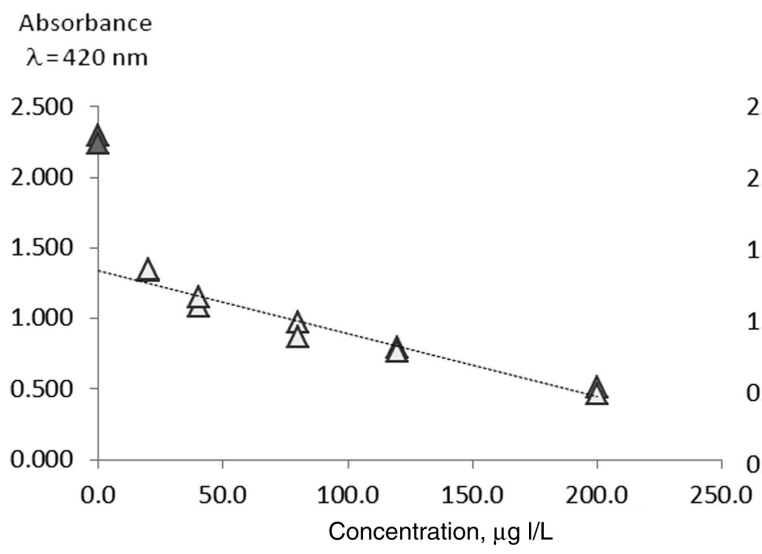

B

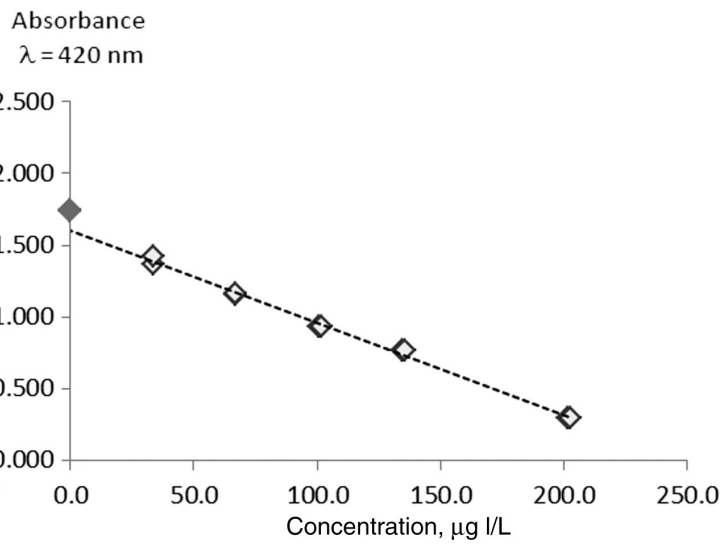

Figure 1: Comparison of calibration curves obtained with the former method (A) and the optimized procedure (B). 
Table 1: Accuracy assessment by comparison of the results obtained with the optimised method and the reference values of the inter-laboratory EQUIP program from Center for Disease Control and Prevention in Atlanta, USA, (CDC).

\begin{tabular}{|c|c|c|c|c|c|c|c|c|}
\hline \multirow[t]{2}{*}{ Sample ID } & & \multicolumn{2}{|c|}{ Our method } & \multicolumn{2}{|r|}{ CDC } & \multicolumn{3}{|c|}{ Mean all laboratories } \\
\hline & & $\mu g \mathrm{I} / \mathrm{L}$ & SD & $\mu g \mathrm{I} / \mathrm{L}$ & RD & $\mu \mathrm{g} \mathrm{I} / \mathrm{L}$ & SD & RD \\
\hline \multirow[t]{3}{*}{ Round 38} & \#1 & 10.5 & 1.2 & 9.33 & $13 \%$ & 10.8 & 5.1 & $-3 \%$ \\
\hline & $\# 2$ & 67.2 & 2.8 & 65.7 & $2 \%$ & 65.3 & 14.0 & $3 \%$ \\
\hline & \#3 & 142 & 2 & 138 & $3 \%$ & 137 & 26 & $3 \%$ \\
\hline \multirow[t]{3}{*}{ Round 39} & \#4 & 85.6 & 1.8 & 89.2 & $-4 \%$ & 87.7 & 13.8 & $-2 \%$ \\
\hline & $\# 5$ & 390 & 4 & 437 & $-11 \%$ & 424 & 45 & $-8 \%$ \\
\hline & \#6 & 190 & 5 & 184 & $3 \%$ & 182 & 22 & $4 \%$ \\
\hline \multirow[t]{4}{*}{ Round 40} & $\# 7$ & 235 & 14 & 245 & $-4 \%$ & 235 & 32 & $0 \%$ \\
\hline & $\# 8$ & 66.4 & 1.4 & 64.5 & $3 \%$ & 64.3 & 11.1 & $3 \%$ \\
\hline & $\# 9$ & 12.4 & 1.1 & 11.8 & $5 \%$ & 12.8 & 5.9 & $-3 \%$ \\
\hline & $\# 10$ & 113 & 2 & 104 & $9 \%$ & 99.7 & 16.1 & $13 \%$ \\
\hline \multirow[t]{3}{*}{ Round 41} & \#11 & 416 & 7 & 399 & $4 \%$ & 385 & 31 & $8 \%$ \\
\hline & $\# 12$ & 389 & 11 & 359 & $8 \%$ & 350 & 40 & $11 \%$ \\
\hline & $\# 13$ & 150 & 11 & 137 & $10 \%$ & 136 & 17 & $11 \%$ \\
\hline \multirow[t]{4}{*}{ Round 42} & \#14 & 95.4 & 3.2 & 89.2 & $7 \%$ & 88.3 & 14.6 & $8 \%$ \\
\hline & $\# 15$ & 256 & 8 & 242 & $6 \%$ & 243 & 28 & $5 \%$ \\
\hline & $\# 16$ & 363 & 26 & 359 & $1 \%$ & 355 & 36 & $2 \%$ \\
\hline & \#17 & 15.8 & 0.4 & 17.2 & $-8 \%$ & 15.9 & 4.1 & $-1 \%$ \\
\hline \multirow[t]{3}{*}{ Round 43} & $\# 18$ & 149 & 2 & 148 & $1 \%$ & 139 & 13 & $7 \%$ \\
\hline & \#19 & 330 & 8 & 307 & $8 \%$ & 297 & 26 & $11 \%$ \\
\hline & $\# 20$ & 69.0 & 1.8 & 71.9 & $-4 \%$ & 67.0 & 9.4 & $3 \%$ \\
\hline
\end{tabular}

$\mathrm{SD}$, standard deviation; RD, relative deviation.

inter-laboratory study of the Center for Disease Control and Prevention (CDC), Atlanta, GA, USA, and the relative deviations were calculated (Table 1).

The results proved to be comparable to the CDC results, with a relative deviation average of $5.7 \%(n=20)$, and $5.4 \%(n=20)$ from the mean of all laboratories in the inter-laboratory program.

In fact, no RSD above $10 \%$ was observed, and only a minor percentage of samples $(12 \%)$ had a relative deviation $>5 \%$. In overall, $88 \%$ of the samples had relative deviations $<5 \%$.

The improved method was comparable with previously described procedures, using the same reaction (Supplemental Table 2). It presented a limit of detection below most of the previously described works and consequently a lower dynamic application range. The RSD for the blank of $2 \%(n=18)$ is a clear evidence of the robustness and reliability of the improved method. The only potential drawback was the determination rate, as it depends upon the digester capacity, due to the $1-\mathrm{h}$ incubation period required. Overall, the above-mentioned advantages overpower the disadvantages, making this improved procedure a valuable innovation to the former method.

Acknowledgments: The authors thank the CDC (Centers for Disease Control and Prevention, Atlanta, USA) for providing the urine samples and reference results.

Author contributions: All the authors have accepted responsibility for the entire content of this submitted manuscript and approved submission.

Research funding: This research was partially supported by the Strategic Funding UID/Multi/04423/2013 and UID/ Multi/50016/2013 through national funds provided by FCT - Foundation for Science and Technology and European Regional Development Fund (ERDF), in the framework of the program PT2020.

Employment or leadership: None declared.

Honorarium: None declared.

Competing interests: The funding organization(s) played no role in the study design; in the collection, analysis, and interpretation of data; in the writing of the report; or in the decision to submit the report for publication.

\section{References}

1. World Health Organization, Assessment of iodine deficiency disorders and monitoring their elimination. A guide for programme managers, 3rd ed., 2008. Available at: http://www.who.int/nutrition/publications/micronutrients/ iodinedeficiency/9789241595827/en/index.html. Accessed: 14 Nov 2016.

2. Khazan M, Azizi F, Hedayati M. A review on iodine determination methods in salt and biological samples. Scimetr 2013;1:e12965.

3. Zimmermann MB. Iodine deficiency. Endocr Rev 2009;30: 376-408.

4. Shelor CP, Dasgupta PK. Review of analytical methods for the quantification of iodine in complex matrices. Anal Chim Acta 2011;702:16-36.

5. Jooste PL, Strydom E. Methods for determination of iodine in urine and salt. Best Pract Res Clin Endocrinol Metab 2010;24:77-88.

6. Sandell EB, Kolthoff IM. Micro determination of iodine by a catalytic method. Microchi Acta 1937;1:9-25.

7. Bland JM, Altman DG. Statistical methods for assessing agreement between two methods of clinical measurement. Lancet 1986;327:307-10.

Supplemental Material: This article contains (DOI:10.1515/cclm2016-1094) offers supplementary material, available to authorized users. 